Methods NEFRORED begins in 2008 as a multicenter registry of prospective observational cohorts, which includes patients with confirmed diagnosis of NL through renal biopsy. Study the incidence of the clinical and pathological characteristics of NL in the Colombian Caribbean region. The database was implemented with the variables of the clinical records of the patients.

Results Eight centres were involved and 400 patients have been included, $88 \%$ female; ratio between men and women is 7:1 with a median age 37 years. Urinary parameters have evidenced 24 hours mean proteinuria of $1.9 \pm 3.3 \mathrm{~g} / 24$ hours and microscopic hematuria in 50\% of patients. Among nephrological syndromes, nephritic syndrome and hematuria-proteinuria have been the most frequent $70 \%$, followed by nephrotic syndrome and non-nephrotic proteinuria 30\%. Hypocomplementemia is evidenced in $60 \%$ of the patients. The median of the SLEDAI has been 10.2 points. Antinuclear Antibodies are positive in $90 \%$. NL class IV-G (A) has been the most frequent $75 \%$. Chronic Renal Disease is concomitant in $52 \%$ of the cases.

Conclusions NEFRORED represents the first Colombian cohort of patients diagnosed with NL, with up-to-date information on the renal manifestations of SLE, comorbidities, disease status and clinical evolution under conditions of real clinical practice. CKD remains a significant comorbidity in patients with NL.

\section{USE OF TICS FOR THE GENERATION OF A MODEL OF MANAGEMENT AND FOLLOW-UP OF PATIENTS WITH LUPIC NEPHRITIS}

${ }^{1,2} \mathrm{G}$ Aroca Martínez, ${ }^{1,2} \mathrm{~A}$ Cadena Bonfanti, ${ }^{1} \mathrm{H}$ Gonzalez Torres* ${ }^{3} \mathrm{~K}$ Moreno, ${ }^{3} \mathrm{M}$ Garay Palacio, ${ }^{3} \mathrm{G}$ Palacio Betancourt. 'Universidad Simon Bolivar, Medicine, Barranquilla, Colombia; ${ }^{2}$ Clinica de la Costa, Nephrology, Barranquilla, Colombia; ${ }^{3}$ TIC Social, Project, Bogota D.C., Colombia

\subsection{6/lupus-2017-000215.398}

Background and aims Our objective was to create a model for the characterisation, classification and management, according to the protocols, of patients with lupus nephritis, which is done in real time and with the generation of alerts at the right time, which allows inferring that we will obtain a Better quality of life of the patients, reduction of complications and, therefore, decrease in the cost of handling these patients.

Methods Initially patients with NL were classified with the CCM system for the risk of the disease. With the help of the CCM information tools, protocols of care and follow-up were incorporated into the software, which made it possible to manage, monitor and control patients.

Results The management of NL patients was handled by a group of specialists whose ultimate goal was to improve the quality of life and identify possible deterioration in the patient's health. In an integral way with CCM, a work plan was determined for each of them, keeping a strict record of the results of examinations that allowed to take medical actions in favour of the patients. Also, a mobile application (CheckUp!) is made available to the user in order to monitor the patient in real time with NL.
Conclusions It was established a flow of actions to manage alterations in the quality of life of the patient. Actions that led to the change in the risk category of the disease per patient and a greater adherence and incorporation to the management to the family nucleus, making the model a success.

\section{THE RELATIONSHIP BETWEEN SOCIOECONOMIC FACTORS AND IMMUNOSUPPRESSIVE USE IN SYSTEMIC LUPUS ERYTHEMATOSUS}

${ }^{1} \mathrm{~A}$ Bankole*, ${ }^{2} \mathrm{~S}$ Ford, ${ }^{2} \mathrm{~F}$ Wong, ${ }^{2} \mathrm{~J}$ McMunn, ${ }^{2} \mathrm{SS}$ Zaman, ${ }^{2} \mathrm{~J}$ Rawlins. ${ }^{1}$ Carilion Clinic, Rheumatology, Roanoke, USA; ${ }^{2}$ Carilion Clinic, Internal medicine, Roanoke, USA

\subsection{6/lupus-2017-000215.399}

Background and aims Systemic Lupus Erythematosus (SLE) is more common in minorities, who also tend to be more socially disadvantaged. Some studies have shown a disparity in access to care and medication prescribed in minorities and socially disadvantaged people with SLE. The purpose of this study was to examine the association between socioeconomic factors including gender, race, and median household income and medication prescribed in our SLE cohort.

Methods The study design was a retrospective cohort single centre study. A review of electronic medical record yielded 115 patients that were included in the cohort. General demographic information including age, gender, zip code and serological testing was obtained through chart review and recorded in the database. Supplemental socioeconomic information for each zip code in Southwest Virginia was obtained from the United States Government Census website. The data was analysed by Kruskal-Wallis Test, Mann-Whitney U Test using SAS9.3.

Results Among the 113 patients, there was no relationship between socioeconomic factors and medication prescribed. There was no statistical relationship between gender and medication prescribed. There was no statistical relationship between race and medications. More importantly, no statistical relationship exists between median household income and medications prescribed.

Conclusions There was no relationship between socioeconomic factors and the medications prescribed in SLE within our cohort. This may relate to treatment standardisation of SLE and the increasing awareness of the "treat to target" ethos in rheumatology. The limitations of this study include the small sample size and it's restriction to a single centre.

\begin{tabular}{|c|c|c|}
\hline \multicolumn{2}{|c|}{ Sex and Racial Distribution } \\
\hline & $N$ & Percent \\
\hline Female & 95 & $87.2 \%$ \\
\hline male & 14 & $12.8 \%$ \\
\hline Caucasian Americans & 73 & $67.0 \%$ \\
\hline African Americans & 28 & $25.7 \%$ \\
\hline Hispanic Amercains & 2 & $1.8 \%$ \\
\hline Other (not included in & 6 & $5.5 \%$ \\
\hline
\end{tabular}

